Pathologe 2008 $29: 301-304$

DOI 10.1007/s00292-007-0951-5

Online publiziert: 14. Oktober 2007

(c) Springer Medizin Verlag 2007
A. Zimpfer ${ }^{1,2} \cdot$ M. Wasner ${ }^{3} \cdot$ F. Fend ${ }^{4} \cdot$ M. Tolnay ${ }^{1} \cdot$ S. Dirnhofer ${ }^{1}$

${ }^{1}$ Institut für Pathologie, Universitätsspital Basel, Schweiz

${ }^{2}$ Gemeinschaftspraxis für Pathologie Prof. Koch, Dr. Hellerich, Dr. Venzke, Freiburg

${ }^{3}$ Department für Neurochirurgie, Universitätsspital Basel, Schweiz

${ }^{4}$ Institut für Pathologie der Technischen Universität, München

\section{Primäres zerebelläres T-Zell-Lymphom}

lär durchgeführt. Intraoperativ zeigte sich eine vom Kleinhirnparenchym nicht abgrenzbare, diffus infiltrierende Raumforderung. Zur histopathologischen Untersuchung wurden mehrere, beige-weiße und rötliche, weiche Gewebestücke von $85 \times 80 \times 10 \mathrm{~mm}$ eingesandt. Histologisch zeigte sich Kleinhirngewebe mit ausgedehnten lymphoiden Infiltraten, begleitet von Nekrosearealen und Einblutungen. Das lymphoide Infiltrat bestand aus vorwiegend kleinen Lymphozyten mit teilweise irregulären, unregelmäßig eingefalteten Zellkernen, vermischt mit einigen mittelgroßen Zellen und ganz spärlich blastären Zellelementen. Daneben fand sich eine massive Vermehrung von Histiozyten. Auffällig war die deutliche Angiozentrizität des Infiltrats ( $\bullet$ Abb. 2 a) und zusätzlich auch eine gewisse Angiodestruktion mit der Folge der beschriebenen Nekrosen. In histologischen Routinefärbungen (Pas, Ziehl-Neelsen, Grocott) ergaben sich keine Hinweise auf spezifische Erreger.

\section{Immunhistochemie}

Immunhistochemisch zeigten die lymphoiden Zellen eine kräftige, homogene Expression von $\mathrm{CD}_{3}, \mathrm{CD}_{5}$ und $\mathrm{CD}_{45} \mathrm{RO}$ (- Abb. 2 b). Die T-Lymphozyten exprimierten zum größten Teil CD4. CD8 wurde lediglich von wenigen reaktiven T-Zellen exprimiert. Die Terminaledesoxynucleotidyltransferase (TdT) war negativ. CD2o war in wenigen B-Zellen positiv. Daneben gab es zahlreiche CD68positive Histiozyten. Der Mib1-Proliferationsindex betrug bis zu $40 \%$. Ein immunhistochemischer Nachweis des latenten Membranantigen 1 (LMP-1) des EpsteinBarr-Virus (EBV) war negativ.

\section{Molekularbiologie}

Eine In-situ-Hybridisierung für „EpsteinBarr encoded small RNA“ (EBER) zeigte eine Positivität in maximal 10\% aller Zellen. Die durchgeführte Polymerasekettenreaktion (PCR) und Fragmentlängenanalyse zum Nachweis einer klonalen T-ZellRezeptor- (TZR-) $)_{\text {gamma }}$-Gen-Umlagerung

\section{Bildgebung}

In der zerebralen Computertomographie (CCT) stellten sich in der rechten Kleinhirnhemisphäre 2 primär metastasenverdächtige, randständig hyperdense, mit Kontrastmittel randständig anreichernde Läsionen mit perifokalem Ödem, Impression des 4. Ventrikels und beginnendem Hydrocephalus occlusivus (• Abb.1) dar. Dadurch war die Indikation zur Kraniotomie gegeben.

\section{Histologie}

Es wurde eine subokzipitale Kraniotomie mit Tumorresektion rechts zerebel-
Abb. 1 - CCT mit Nachweis von 2 randständig hyperdensen und kontrastmittelaufnehmenden (rechts) Läsionen im rechten Zerebellum

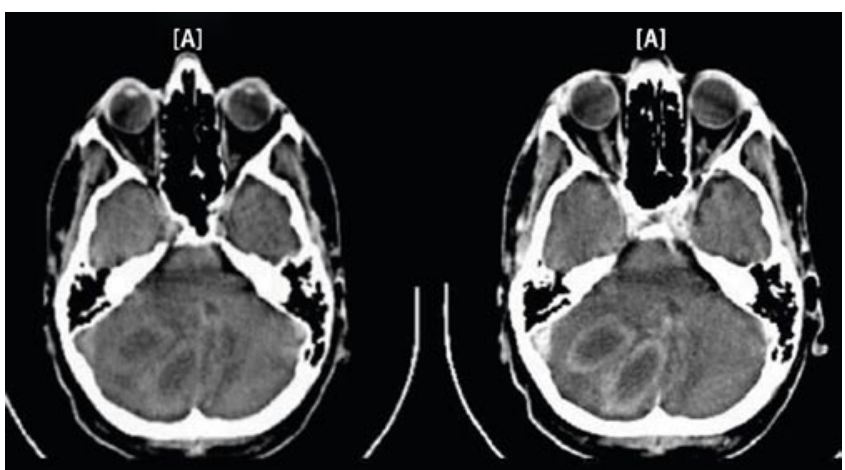




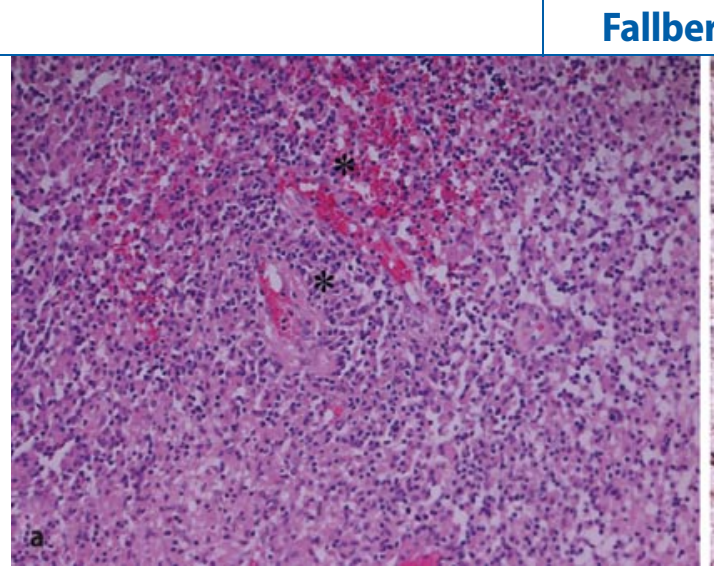

\section{richte}

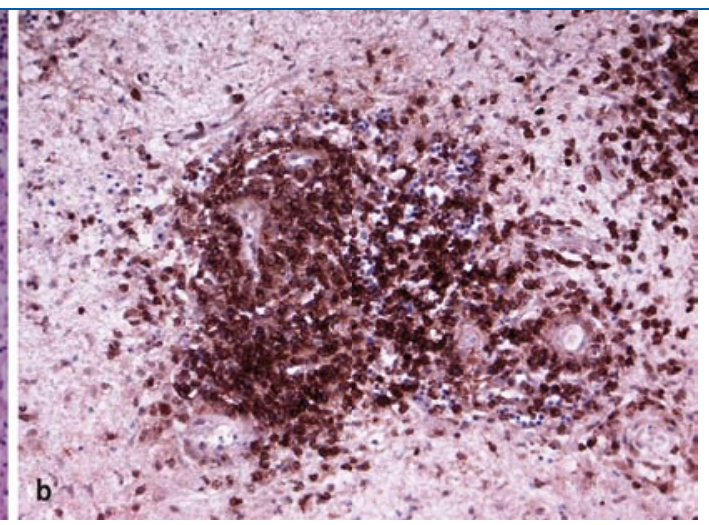

Abb. $2<$ Histologie des primären T-Zell-Lymphoms im Kleinhirn. a Es finden sich atypische lymphoide Infiltrate, Angiodestruktionen $\left({ }^{*}\right)$, Nekrosen und Blutungen.(HE-Färbung, Vergr. 1:100) b Starke und homogene Expression des Antigens CD4. Darstellung der Angiozentrizität des malignen T-Zell-Infiltrats. CD4-Immunhistochemie (IHC), Vergr. 1:200)

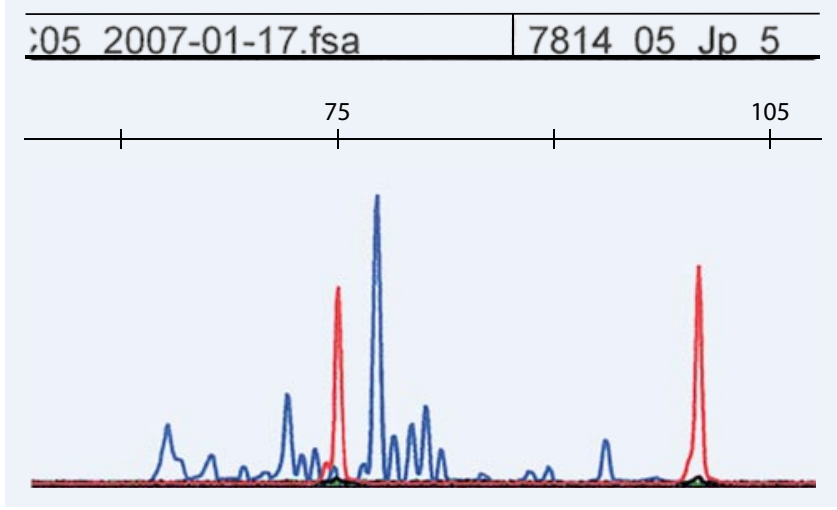

Abb. $3<$ Nachweis einer klonalen TZR-GenUmlagerung in der Fragmentlängenanalyse nach PCR. Es zeigt sich eine $5 f a c h$ erhöhte klonale Bande bei 78 bp mit dem JpPrimer-Set zeigte eine um das 5 fache erhöhte klonale Bande bei 78 bp (• Abb. 3). Die Diagnose eines peripheren T-Zell-Lymphoms, „not other specified“ (NOS), mit Manifestation im Kleinhirn wurde damit abschließend gestellt.

\section{Therapie und Verlauf}

Nach der Operation kam es zu keinen progredienten neurologischen Ausfällen. Eine postoperativ durchgeführte CCT zeigte eine gute Entlastung, konnte einen Resttumor aber nicht ausschließen. StagingUntersuchungen ergaben keine eindeutigen Hinweise auf eine extradurale Lymphommanifestation. Der Patient erhielt Methotrexat (MTX, Hochdosistherapie, nur intravenöse Gabe, $4 \mathrm{~g} / \mathrm{m}^{2}$ ) jeweils am Tag 1 in 2-wöchigen Zyklen. Eine vor Gabe des 3. Zyklus durchgeführte MRT des Schädels zeigte keine residualen Tumoranteile. Die 3. Zyklusgabe MTX unterblieb wegen Thrombopenie.

Im weiteren Verlauf entwickelte der Patient septische Temperaturen und Atemnot. Eine Pneumocystis-carinii-Pneumonie wurde diagnostiziert. Der Zustand des Patienten verbesserte sich im Verlauf von
3-4 Wochen. Er war unter intermittierender druckkontrollierter „Bilevel positive airway pressure-“ (BiPAP-)Beatmung und Sauerstoffgabe respiratorisch stabil. Allerdings trat 10 Wochen nach Diagnosestellung der Tod im Rahmen eines plötzlichen Herz-Kreislauf-Stillstandes bei Kammerflimmern ein. Die durchgeführte Autopsie zeigte eine chronische organisierende Pneumonie aller Lungenlappen ohne Nachweis von Pneumocystis carinii.

Die Hirnsektion ergab einen postoperativen rechtszerebellären $30 \times 20 \times 20 \mathrm{~mm}$ messenden Substanzdefekt. Im Zerebellum war kein Residualtumor nachweisbar. Weder im restlichen zentralen Nervensystem (ZNS) noch in anderen Organsystemen ergaben sich Hinweise für eine weitere Lymphommanifestation.

\section{Diskussion}

Primäre Lymphome des ZNS (PZNSL) sind mit $1-2 \%$ aller Non-HodgkinLymphome selten [8]. In der Regel handelt es sich um B-Zell-Lymphome [1]. Ein primäres T-Zell-Lymphom des ZNS (PTZL ZNS) fand sich in $2-8,5 \%$ groß- er ZNS-Lymphom-Fallserien [1, 8]. Die PTZL ZNS treten bei Immunsupprimierten und bei Patienten ohne bekannten Immundefekt auf $[4,5]$. Es sind mehr Männer als Frauen betroffen.

Die Literaturdaten zeigen einen Lymphombefall des Hirnstamms, Zerebellum und selten auch des Rückenmarks in etwa $1 / 3$ der Fälle und einen Befall des Großhirns in $2 / 3$ der PTZL ZNS. Eine bessere Prognose der PTZL ZNS mit niedriggradiger Morphologie wurde diskutiert, aber in der bisher größten Serie von 45 Fällen als nicht signifikant bewertet [8].

Da das PTZL ZNS des immunkompetenten Patienten ein sehr seltenes Ereignis ist, fehlen im Gegensatz zu PTZL ZNS im Rahmen einer Immunsuppression oder auch im Gegensatz zu den „häufigeren“ BZell-Lymphomen des ZNS hier klare Therapiekonzepte. Die immunkompetenten Patienten mit PTZL ZNS werden im Rahmen von Studien mit B-Zell-Lymphomen des ZNS geführt und in den meisten Fällen mit einer Hochdosis-MTX-Therapie oder MTX-basierten Polychemotherapie behandelt $[5,8]$. Die Überlebensraten beziehen sich somit auf die gesamte Population der PZNSL bei immunkompetenten Patienten mit primär gutem Ansprechen von $2 / 3$ der Patienten auf die durchgeführte MTX-Therapie. Patienten dieser Gruppe erleiden jedoch fast durchweg innerhalb von 2-5 Jahren ein Rezidiv und versterben im Vergleich früher als Patienten mit systemischem Befall durch ein großzelliges B-Zell-Lymphom [5].

Die Pathogenese der PZNSL - sowohl der B- als auch der T-Zell-Lymphome ist ungeklärt. Eine Hypothese geht davon aus, dass ein maligner lymphoider Zellklon mittels speziellen Adhäsionsmolekülen das Gehirn besiedelt (zitiert und 
besprochen in $[2,5])$. Bei immunsupprimierten Patienten wurde eine Infektion mit dem EBV in 50-100\% der Fälle mit BZell-Phänotyp nachgewiesen $[3,6]$, was eine infektiöse Ätiologie wahrscheinlich macht. Zu den PZNSL mit T-Phänotyp gibt es diesbezüglich keine größeren Studien. Bei nichtimmunsupprimierten $\mathrm{Pa}-$ tienten sind nur wenige Fälle mit einer EBV-Infektion oder eine Infektion mit dem humanen T-lymphotrophischen Virus $1(\mathrm{HTLV}-1)$ beschrieben worden $[4,8]$. Wir fanden in unserem Fall nur vereinzelte EBER-positive, LMP1-negative Zellen, was für reaktive „Bystander-Lymphozyten" und gegen eine kausalpathogenetische Rolle von EBV spricht.

\section{Differenzialdiagnose mittels Immunhistochemie}

Mittels Immunhistochemie können die meisten PTZL ZNS von anderen Entitäten wie B-Zell-Lymphomen, primären oder sekundären ZNS-Tumoren wie u. a. High-grade-Gliomen, Karzinommetastasen, dem malignen Melanom und primitiven neuroektodermalen Tumoren differenziert werden.

Allerdings ist die histopathologische und immunhistochemische Untersuchung gerade bei niedriggradiger Morphologie wie im vorliegenden Fall nicht immer ausreichend, um ein PTZL ZNS sicher von entzündlichen demyelinisierenden Läsionen, Vaskulitiden, viralen oder bakteriellen Schädigungen sowie von diffus großzelligen B-Zell-Lymphomen des ZNS mit zahlreichen reaktiven TLymphozyten zu differenzieren $[3,7,10]$. In einigen Fällen wurde primär aufgrund der erhöhten Lymphozytenzahl in der Liquorpunktionsflüssigkeit die Verdachtsdiagnose einer viralen Meningoenzephalitis gestellt und erst nach zeitintensivem Ausschluss viraler, bakterieller und fungaler Infektionen (einschließlich Herpessimplex-Virus, Zytomegalievirus, Varizella-Zoster-Virus, EBV, HIV, Borrelien und Toxoplasma gondii) der Weg zur Diagnose eines PTZL ZNS verfolgt [9].

Bei klinischem Lymphomverdacht sollte, um Zeitverzögerungen zu vermeiden, eine frühe Hirnbiopsie angestrebt werden. Gezielte immunhistochemische Untersuchungen, inklusive T-Zell-Marker,
NK-Zell-Marker und Marker für zytotoxische Proteine, wie auch der Nachweis einer monoklonalen $T Z R_{\text {gamma }}-G e n-$ Umlagerung sollten zur Differenzierung der PTZL ZNS von inflammatorischen Läsionen oder auch Unterscheidung zwischen T-Zell-reichen diffusen großzelligen B-Zell-Lymphomen vom PTZL ZNS zum diagnostischen Standard gehören.

\section{Fazit für die Praxis}

Die Diagnose eines primären T-ZellLymphoms des ZNS erfordert eine komplexe Diagnostik mit gezielter Immunhistochemie und dem Nachweis einer klonalen TZR-Gen-Umlagerung zum Ausschluss eines reaktiven, entzündlichen Prozesses.

Die Differenzialdiagnose bleibt aber besonders bei Vorliegen nur kleiner hämorrhagischer Gewebefragmente schwierig. Die Integration der klinischen Angaben und der Bildgebung mit der Histologie, Immunohistochemie und molekulargenetischen Daten ist deshalb in diesen Fällen essenziell.

\section{Korrespondenzadresse \\ Prof. Dr. S. Dirnhofer}

Institut für Pathologie, Universitätsspital Basel Schönbeinstraße 40, 4031 Basel

sdirnhofer@uhbs.ch

Interessenkonflikt. Der korrespondierende Autor gibt an, dass kein Interessenkonflikt besteht.

\section{Literatur}

1. Burke JS (2001) Waldeyer's ring, sinonasal region, salivary gland, thyroid gland, central nervous system, and other extranodal lymphomas and lymphoid hyperplasia. In: Knowles DM (ed) Neoplastic haematopathology. Lippincott Wiliams \& Wilkins, Baltimore, pp 1370-1372

2. Ferreri AJM, Abrey LE, Blay JY et al. (2003) Summary statement on primary central nervous system lymphomas from the Eighth International Conference on Malignant Lymphoma, Lugano, Switzerland, June 12 to 15, 2002. J Clin Oncol 21: 24072414

3. Grant JW, Isaacson PG (1992) Primary central nervous system lymphoma. Brain Pathology 2: 97109

4. Hayabuchi N, Shibamoto Y, Onizuka Y et al. (1999) Primary central nervous system lymphoma in Japan: a nationwide survey. Int J Radiat Oncol Biol Phys 44: 265-272

5. Hochberg FH, Baehring JM, Hochberg EP (2007) Primary CNS lymphoma. Nat Clin Prac Neurol 3: 24-35
Pathologe 2008 $\cdot 29: 301-304$

DOI 10.1007/s00292-007-0951-5

○) Springer Medizin Verlag 2007

A. Zimpfer · M. Wasner · F. Fend · M. Tolnay · S. Dirnhofer

Primäres zerebelläres

T-Zell-Lymphom

Zusammenfassung

Primäre T-Zell-Lymphome des zentralen Nervensystems (ZNS) sind selten. Sie müssen differenzialdiagnostisch von reaktiven Läsionen unterschieden werden. Die Diagnosestellung sollte integrativ unter Verwendung von immunhistochemischen, molekulargenetischen und/oder zytogenetischen Methoden erfolgen. Wir beschreiben den Fall eines 50 -jährigen Mannes, bei welchem ein primäres zerebelläres T-Zell-Lymphom diagnostiziert und eine klonale T-Zell-Rezeptorgen-Umlagerung nachgewiesen wurde. Nach 2 Zyklen Chemotherapie entwickelte der Patient eine Pneumozystis-carinii-Pneumonie und verstarb 10 Wochen nach Diagnosestellung. Die Autopsie ergab keinen Residualtumor im ZNS.

Schlüsselwörter

Extranodale Non-Hodgkin-Lymphome . T-Zell-Lymphom · ZNS-Lymphom

\section{Primary cerebellar T-cell lymphoma}

Abstract

Primary central nervous system T-cell lymphomas are rare and have to be differentiated from reactive lesions. It is therefore essential to use all possible tools to establish the diagnosis, including immunohistochemistry, molecular genetic analysis, and/or cytogenetic methods. In this paper we present the case of a primary cerebellar T-cell lymphoma in a 50-year-old man; a clonal T-cell receptor gene rearrangement was documented. After two cycles of methotrexate therapy the patient developed Pneumocystis cariniiinduced pneumonia, dying 10 weeks after his diagnosis. The autopsy did not reveal any residual tumour.

Keywords

Extranodal non-Hodgkin lymphoma · T-cell lymphoma - CNS lymphoma 


\section{Service}

6. Jellinger KA, Paulus W (1995) Primary central nervous system lymphomas - new pathological developments. J Neurooncol 24: 33-36

7. Postler E, Bornemann A, Skalej M et al. (1999) Intracranial inflammatory tumors: a survey of their various etiologies by presentation of 5 cases. J Neurooncol 43: 209-217

8. Shenkier TN, Blay J, O,Neill BP et al. (2005) Primary CNS lymphoma of T-cell origin: a descriptive analysis from the international Primary CNS Lymphoma Collaborative Group. J Clin Oncology 10: 22332239

9. Stark AM, Tiemann M, Dörner L et al. (2004) Primary peripheral T-cell lymphoma of the central nervous system. Zentralbl Neurochir 65: 191-194

10. Wanschitz J, Hainfeller JA, Simonitsch I et al. (1997) Non-HTLV-1 associated pleomorphic T-cell lymphoma of the brain mimicking post-vaccinal acute inflammatory demyelination. Neuropathol Appl Neurobiol 23: 43-49

\section{Termine}

Möchten Sie eine Veranstaltung ankündigen? Bitte tragen Sie Ihre Termine auf der KongressSeite von DerPathologe.de ein

Wir freuen uns auf Ihre Veranstaltungshinweise!

\section{August 2008}

Bern, Schweiz 25.-27.08.2008 Virtopsy Basic Course

Auskunft: Frau Sandra Salzmann, Institute of Forensic Medicine, Buehlstrasse 20, CH-3012 Bern, Schweiz,

Fon: +41 (0)31 6318422

Fax: +41(0)3163184 15,

sandra.salzmann@irm.unibe.ch, www.virtopsy.com

Graz, Österreich 25.-27.08.2008

Postgraduate Course Lung, Pleural \&

Mediastinal Diseases

Part I: Common Diseases

Auskunft: Frau Mag. Edith Kleinferchner,

Medizinische Universität Graz,

Auenbruggerplatz 25, 8036 Graz, Österreich,

Fon: 0043 (0) 3163804407

Fax: 0043 (0) 3163809638 ,

edith.kleinferchner@meduni-graz.at,

http://pathologie.meduni-graz.at/Pathologie/ veranstaltungen.htm

Graz, Österreich 28.-30.08.2008

Postgraduate Course Lung, Pleural \&

Mediastinal Diseases

Part II: Rare Diseases

Auskunft: Frau Mag. Edith Kleinferchner,

Medizinische Universität Graz,

Auenbruggerplatz 25, $8036 \mathrm{Graz}$, Österreich,

Fon: 0043 (0) 316380 4407,

Fax: 0043 (0) 3163809638 ,

edith.kleinferchner@meduni-graz.at,

http://pathologie.meduni-graz.at/Pathologie/

veranstaltungen.htm

\section{September 2008}

Mainz 08.-10.09.2008

Endomikroskopie-Kurs

Themen: Konfokale Endomikroskopie/

Workshop

Auskunft: Frau Heike Orlando, Pentax Europe

GmbH, Julius-Vosseler-Str. 104, 22527 Hamburg

Fon: 040 56192381,

Fax: 0405604213 ,

orlando.heike@pentax.de,

www.endomicroscopy.com

Wien, Österreich 11.-13.09.2008

Gemeinschaftstagung der Schweizer und Österreichischen Gesellschaft für

Senologie unter Beteiligung der Deutschen Gesellschaft für Senologie

Themen: Brustkrebs im 21. Jahrhundert - Vision und Wirklichkeit

Auskunft: Ärztezentrale Med.Info,

Helferstorferstrasse 4, 1014 Wien, Österreich,

Fon: (+43/1) 53116 - 48,

Fax: (+43/1) $53116-16$,

azmedinfo@media.co.at

www.wenologie.at
Mannheim 21.-24.09.2008

5. Jahrestagung der Deutschen Vereinten Gesellschaft für Klinische Chemie und Laboratoriumsmedizin (DGKL)

Laboratory Diagnostics: From Bench to Bedside

Themen: Klinische Chemie, Laboratoriumsmedizin

Auskunft: Conventus Congressmanagement \& Marketing GmbH, Markt 8, 07743 Jena, Fon: +49 (0)3641 35330 , Fax: +49 (0)364135332 71, www.conventus.de

\section{Oktober 2008}

Graz, Österreich 08.-10.10.2008 Postgraduate Course Diagnostic Breast Pathologie

Auskunft: Frau Mag. Edith Kleinferchner,

Medizinische Universität Graz

Auenbruggerplatz 25, 8036 Graz, Österreich, Fon: 0043 (0) 3163804407 ,

Fax: 0043 (0) 3163809638

edith.kleinferchner@meduni-graz.at, http://pathologie.meduni-graz.at/Pathologie/ veranstaltungen.htm

Graz, Österreich 27.-28.10.2008

Postgraduate Course Lung, Pleural \&

Mediastinal Diseases

Part III: Mediastinal Diseases

Auskunft: Frau Mag. Edith Kleinferchner,

Medizinische Unversität, Auenbruggerplatz 25 , 8036 Graz, Österreich,

Fon: 0043 (0) 3163804407

Fax: 0043 (0) 3163809638 ,

edith.kleinferchner@meduni-graz.at,

http://pathologie.meduni-graz.at/Pathologie/ veranstaltungen.htm

\section{November 2008}

Mainz 03.-05.11.2008

Endomikroskopie-Kurs

Themen: Konfokale Endomikroskopie/

Workshop

Auskunft: Frau Heike Orlando, Pentax Europe GmbH, Julius-Vosseler-Str. 104, 22527 Hamburg Fon: 04056192381

Fax: 0405604213 ,

orlando.heike@pentax.de,

www.endomicroscopy.com

Berlin 11.11.2008

Berliner Gesellschaft für Pathologie (e.V.)

386. Wissenschaftliche Sitzung

Themen: Mammakarzinom - update

Auskunft: Herr Prof. Dr. Hartmut

Lobeck, Klinikum Ernst von Bergmann,

Charlottenstraße 72, 14467 Potsdam,

Fon: 0331-2416501,

Fax: 0331-2416500,

hlobeck@klinikumevb.de,

www.klinikumevb.de 